\title{
Application of the VEMP test for diagnosing patients with vestibular neuritis
}

\author{
Kalina Madzharova, Ana Beshkova \\ Department of ENT diseases, University hospital Sveti Georgi, \\ Medical University of Plovdiv, Vassil Aprilov Blvd 15 A 4000 Plovdiv
}

\begin{abstract}
Background: The vestibular evoked myogenic potentials test can be applied when diagnosing vestibular disease. This method is used for neurophysiological assessment of the otolithic apparatus of the vestibular system.

Materials and methods: Monitoring changes in the VEMP test of patients diagnosed with vestibular neuritis in the age range from 19 to 60 years. For the cVEMP test, sound stimulation through the headphones or via the bones through vibrations is used to read the status of macula sacculae, the lower vestibular nerve and the vestibulosaccular paths. The oVEMP test records the status of macula utriculae and the upper branch of the vestibular nerve of the contralateral side.

Aim: Monitoring changes in VEMP tests of patients with vestibular neuritis and assessment of obtained results.

Results: During the first examination, the VEMP test of patients diagnosed with vestibular neuritis registered deviations. After the third month, improvement was noted in the subjective complaints and the results of the VEMP test. Changes were seen predominantly in oVEMP.

Conclusions: The VEMP test can be applied to diagnose peripheral vestibular pathology. The VEMP test is an additional method for diagnosing vestibular neuritis.
\end{abstract}

Keywords: VEMP test, vestibular dysfunction,

\section{Introduction}

Vestibular pathology is an important aspect of otorhinolaryngology. There is increasing frequency of peripheral vertigo caused by inner ear disease. The diagnosis of vestibular disease if often a challenging clinical task due to their variety and lack of objectification.

VEMP is a non-invasive method, which uses sounds or vibrations to stimulate the nerve cells of the utriculus or sacculus, and along the path of the vestibuloocular and vestibulocervical reflexes impulses reach musculus obliquus inferior and musculus sternocleidomastoideus, respectively, and vestibular evoked myogenic potentials are registered (1). This is a relatively new test used to evaluate vestibular dysfunction. A distinction is made between cervical VEMP (cVEMP) test examining the sacculus and an ocular VEMP (oVEMP) test examining the utriculus.

VEMP reaction is present in $100 \%$ otologically healthy patients aged up to 40 years and $95 \%$ of these aged 41-50 years. With electrophysiological measurements, symmetry of readings from both ears is important. The lack of symmetry can be a sign of unilateral vestibular dysfunction. The VEMP and VHIT methods test all main parts of the vestibular system: the three semicircular canals, otoliths, the lower vestibular nerve and upper vestibular nerve on each side. 
The VEMP test is an objective method for evaluation of the otolithic receptors and vestibular pathways. It assesses vestibular function through the reaction of the reflex muscle in response to highintensity acoustic stimulation. Together with other neurological tests, it aids in diagnosing a number of vestibular disorders and neurological diseases, as well as their subsequent preventive care.

Vestibular neuritis is one of the most common causes of peripheral vestibular vertigo. Acute peripheral vestibulopathy occurs with viral infections, vascular, toxic or autoimmune pathologies.

The typical symptoms of vestibular neuritis include vertigo, nausea/vomiting, imbalance following unilateral loss of peripheral vestibular function. Vertigo is usually described as rotational and increases significantly with head movement. Patients display spontaneous horizontal rotational nystagmus directed to the lesion side, unilateral caloric hyporeflexia, reduced response of evoked myogenic potentials (VEMP) of the vestibular system during simulation at the affected ear and imbalance with deviation in the opposite direction to the nystagmus. Recovery is achieved through peripheral and central vestibular compensation. Tests demonstrate injury of the upper, lower or both branches of the vestibular nerve. Based on investigations by Goebel et al., in 2001, it was found that the upper branch is affected more often. The upper branch passes through a long and narrower tunnel in the temporal bone. This makes it more sensitive to inflammation (2)

Various parameters are abnormal in the VEMP test of patients with vestibular neuritis (lack of wave on the involved side, increased latency and reduced amplitude of the p13 - n23 complex or the n10 wave on the involved side). When following-up on patients one year later, $71.4 \%$ of these have recovered to normal values in all tests with regression of vestibular symptoms (3). When the entire vestibular nerve is affected, changes are noted in oVEMP and cVEMP tests. When both branches (upper and lower) of the vestibular nerve are affected, it is considered that the spinal ganglion is affected.

cVEMP examines the lower branch of the vestibular nerve. The term IVN (Inferior vestibular neurit) was first proposed by Halmagyi et al. IVN is a subtype of VN (vestibular neuritis). The final diagnosis of IVN is more difficult than SVN. Therefore, it is preferable to also perform a HIT test of the posterior semicircular canal since cVEMP can sometimes be absent in healthy individuals especially with advanced age. The isolated absence of cVEMP needs to be interpreted carefully because it may be the result of poor activation of musculus sternocleidomastoideus. For patients with vestibular neuritis, the VEMP test can show an elongation of the $\mathrm{p} 13$ wave and/or elongation of the $\mathrm{n} 23$ wave, amplitude asymmetry or absent waves (3).

oVEMP reflects the function of the upper branch of the vestibular nerve. Patients with VN have much higher frequency of abnormal oVEMP. Shinet et al. prove that $73.2 \%$ of patients with $\mathrm{VN}$ have abnormal oVEMP. Deviations in oVEMP depend on the degree of vestibular disorders. According to Gionali et al. the upper branch of the vestibular nerve is longer and more susceptible to disruption of conveyance of excitations (2). Significant oVEMP asymmetry reflects predominantly a utricural lesion (4). The ratio in oVEMP is elevated in VN over $35 \%$. VEMP has a role in diagnosing and predicting outcome in patients with vestibular neuritis (2). The combination of cVEMP and oVEMP testing has an advantage for long-term monitoring of patients with VN. The parameters of the two VEMP responses have a high degree of reliability. This is a screening tool for evaluation of the cross-section vestibulo-ocular reflex and ipsilateral sacculocolic reflex, which cuts diagnostic testing time.

If the upper branch of the vestibular nerve is involved, changes are noted in the contralateral oVEMP and preserved ipsilateral cVEMP (5). In individuals with involvement of the lower branch of the vestibular nerve there is lack of response to the cervical VEMP and normal responses for the ocular VEMP (6). The VEMP test may yield normal results, altered or absent responses. In some patients, both branches of the vestibular nerve are affected, while others have involvement only of the upper branch (lesion only of the upper branch) (7). The analysis of responses to the combined cervical and ocular VEMP test shows results which complement the diagnosis of individuals with vestibular hyporeflexia (6). Careful history taking, an otoneurological and a neurological examination are usually sufficient to diagnose vestibular dysfunction.

\section{Aim}

Monitoring changes in VEMP tests of patients with vestibular neuritis and assessment of obtained results. 


\section{Materials}

Changes are monitored in the VEMP test of 42 adult patients diagnosed with vestibular neuritis in the age range from 19 to 60 years over the period between 05.2018 and 09.2019. Twenty-seven people in the monitored age group are women and $15-$ men. The study was performed at the otoneurological medical office of the ENT clinic at UMHAT Sveti Georgi.

\section{Tasks}

1. Following up on the results of the VEMP test in patients with vestibular dysfunction.

2. Comparing the changes in the VEMP test of the first examination against the results obtained at the follow-up examination following treatment.

3. Comparing the results obtained by the otoneurological examination against the results of the VEMP test.

Exclusion criteria - inflammation of the middle ear, reduced hearing, central vertigo.

\section{Methods}

After taking a detailed case medical history and the examination of ENT organs, was performed tone threshold audiometry, tympanometry and the otoneurological examination. The VEMP test was performed following exclusion of conductive hearing impairment.

An eVEMP usb BioMed computer system was used for the vestibular evoked myogenic potentials test. VEMPusb is a comprehensive system for testing otolith organs

When performing the VEMP examination we used an auditory stimulus (click) $10 \mathrm{~ms}$ with an intensity of $110 \mathrm{~dB}$ (over $90 \mathrm{~dB}$ ) and frequency of $500 \mathrm{~Hz}$.
The recorded test results are displayed on the monitor in four diagrams (two for cVEMP left/right and two for oVEMP left/right).

The presence of typical cVEMP (p13 - n23) and oVEMP (n10-p15) is monitored. If the characteristic biphasic wave pattern in oVEMP and cVEMP testing is not seen, the examination is considered as a non-response. The wave latency, amplitude, threshold for appearance of the potential and amplitude symmetry are recorded. The potential amplitude is directly proportional to the degree of muscle tension and respectively the level of EMG activity.

For the cVEMP test, sound stimulation through the headphones or via the bones through vibrations is used to read the status of macula sacculae, the lower vestibular nerve and the vestibulosaccular paths with the aid of the electrodes. The oVEMP test records the status of macula utriculae and the upper branch of the vestibular nerve on the side contralateral to the electrodes.

\section{Results}

In all patients with vestibular neuritis during the first examination a deviation was found in the otoneurological test. A diagnosis was made of peripheral otoneurological syndrome. Following otoneurological examination, a VEMP test was performed (cVEMP and oVEMP).

Fourteen of the examined patients showed deviations in the cVEMP test and the oVEMP examination. In 23 people, alterations were registered only in oVEMP, predominantly atypical waves. In five patients typical curves were registered for cVEMP and oVEMP. Changes were predominantly in the oVEMP test.

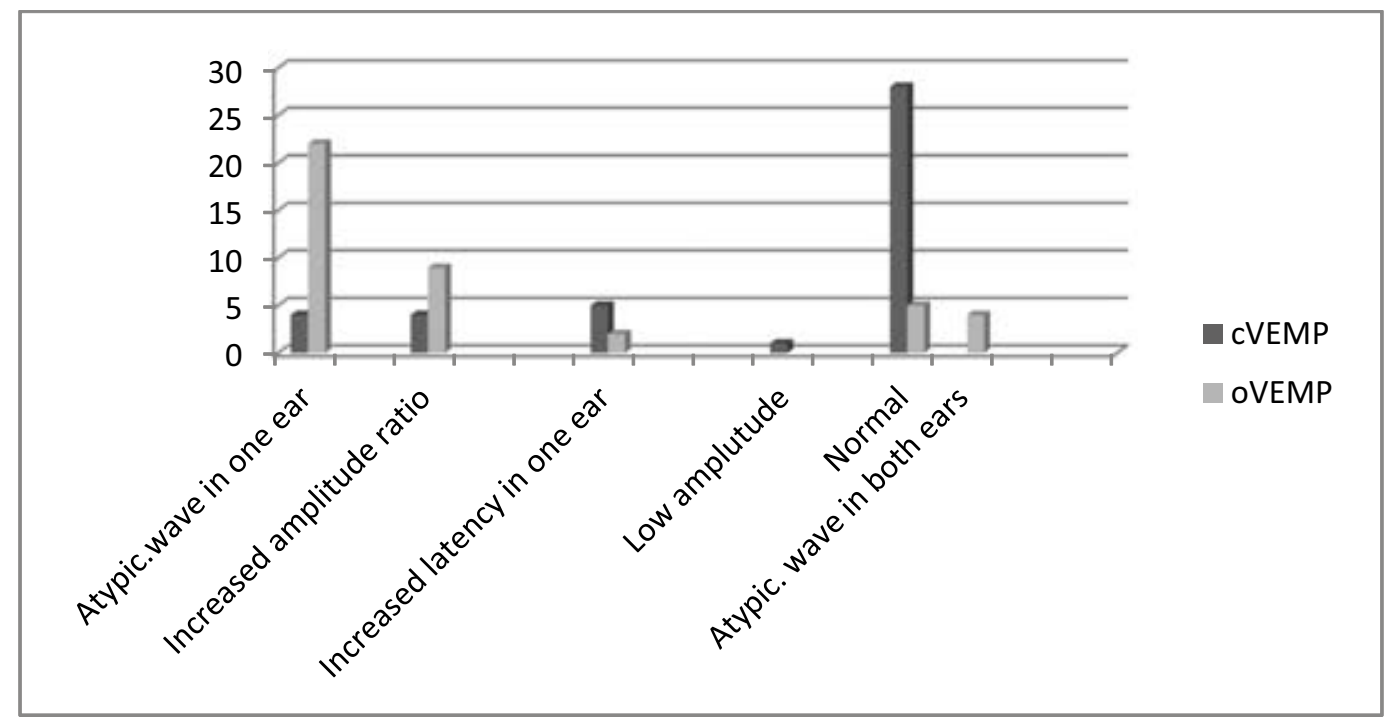


Follow-up examination was conducted three months later. Five of the followed patients did not come in for the follow-up examination. All patients reported improvement of complaints. 37 adult patients underwent otoneurological examination. The otoneurological examinations indicated deviations in eight people with seven of them showing changes also in the oVEMP test.

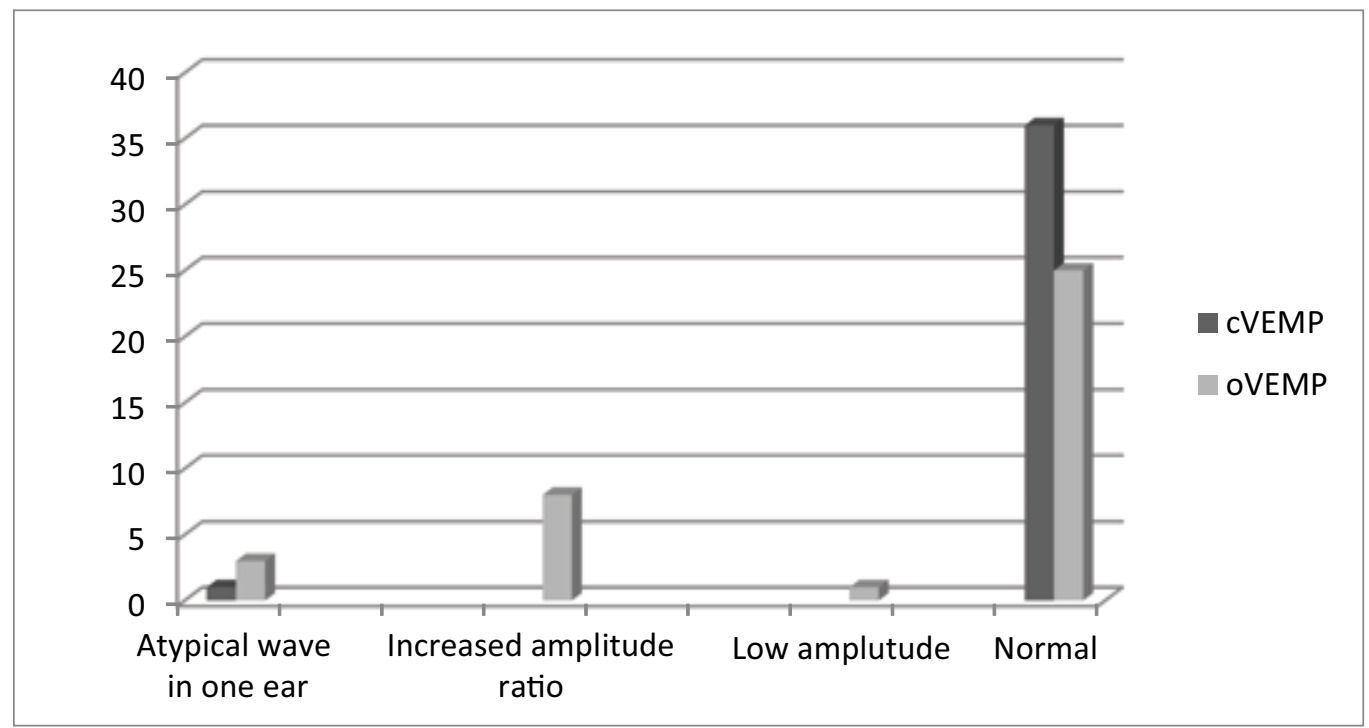

The conducted VEMP test in one of the followed patients registered deviation in the cVEMP and oVEMP test (for cVEMP - atypical wave for one ear, the oVEMP test registered increased amplitude ratio).

The oVEMP test of 25 patients demonstrated typical curves for oVEMP and in 12 -deviations in the oVEMP test. In eight patients, waves with elevated amplitude ratio were registered, three people showed an atypical wave in one ear and one patient had a n-p wave with low amplitude.

After the third month improvement was seen in the results of the VEMP test.

After the third month in 23 of the followed patients there were no deviations in the VEMP test and the otoneurological examination. In 7 patients abnormal curves were registered in the oVEMP test and deviations in the otoneurological examination.

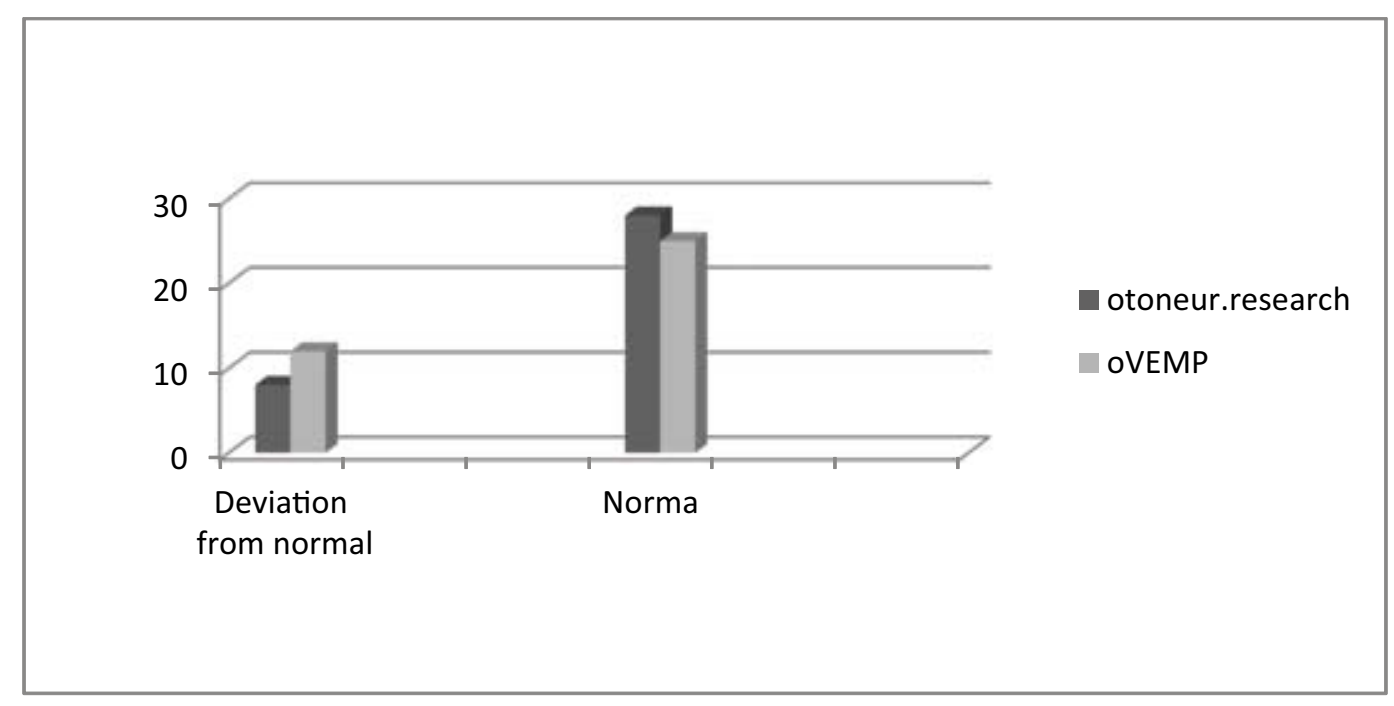


After the third month, seven of the patients had changes registered in the VEMP test, however the otoneurological examination showed no abnormality.

\section{Discussion}

The early diagnosis of vestibular neuritis is often a challenge for the otoneuroligst. A detailed case history and routine otoneurological and neurological methods are sometimes insufficient to provide a precise diagnosis. The VEMP test is an additional method for examining vestibular dysfunction. This test is used to diagnose vestibular disorders and to objectify vestibular symptoms. The combination of cVEMP and oVEMP testing is also advantageous. During the examination of patients with vestibular neuritis deviations were registered in the VEMP test.

In recent years, studies indicate that when diagnosing vestibular neuritis there are predominantly alterations in the oVEMP test (2), (4). When tracking the deviations in the VEMP test of examined patients during their first test and the follow-up one three months later, there were changes predominantly in the oVEMP test.

Three months later, after treatment, improvement was seen both in patient symptoms and the results of the otoneurological examination (3). The results of the VEMP test also showed improvement. Despite the improvement of the subjective status of patients, some of them showed deviations in the VEMP examination. The statistical processing of VEMP test data found significant difference in the results from the third month compared to the beginning.

\section{Conclusion}

The VEMP test can be applied not only for peripheral vestibular pathology but also CNS diseases: migraine-associated vestibulopathy, following head trauma, Herpes zoster oticus, multiple sclerosis, brain tumors, osteochondrosis.

A disadvantage of the VEMP test is that it assesses only partially the status of peripheral vestibular organs. There is an absence of VEMP responses in the case of middle ear effusion, tympanic membrane perforation, and otosclerosis due to weakened stimulus reaching the inner ear. There is difficulty in performing the cVEMP test in patients with cervical disease and muscle weakness. Frequently, cVEMP waves are not registered in people who have undergone neck surgery of those who suffer pain. In the presence of muscle tiredness, only low amplitudes may be recorded. If neck muscles are not activated, VEMP waves are not registered. Age is associated with degenerative changes which affect the vestibular apparatus and central cores.

Practically, there is no one test to assess the entire vestibular apparatus.

The VEMP test is an objective method for examination of the otolith organs of the vestibular system. This is the only method for assessment of the functioning of otolith organs. VEMP has advantages: It is a non-invasive method and is patient-friendly. This test has become part of clinical practice in recent years. The VEMP test can be used in central or peripheral vestibular disorders as a complimentary diagnostic method. This method can be incorporated in the routine clinical otoneurological examination.

\section{References}

1. GuangWei Z, Cox C Vestibular Evoked Myogenic Potentials History and Overview American Journal of Audiology. 2004 Dec Vol. 13(2), 135-143. PMID: 15903139 DOI: 10.1044/1059-0889(2004/018)

2. Adamec I, Skoris KM, Handzic J and al. The Role of Cervical and Ocular Vestibular -EvokedMyogenic Potential in the Follow up of Vestbular Neuritis Clinical EEG and Neuroscience 2014 Vol. 45 (2 ) 129-136

3. Serra PA, Dorigueto SR, Robiero de Almeida R Vestibular myogenic potential in unilateral vestibular hypofunction Acta Oto-Laryngologica 2012 Mar 11; Issue 7 pages 732-738. https://doi.org/10.3109/00016489.2012.659283

4. Rauch S. Vestibular evoked myogenic potentials; Curr Opin Otolaryngol Head Neck Surg 2006 14(5): 299-304 PMID: 16974141 DOI: 10.1097/01.moo.0000244185.65022.01

5. Curthoys SI,Vedran Vulovic, Burgess MA, Leonardo Manzari L, and al. Neural basis of new clinical vestibular test: otolithicneural responses to sound and vibration Clinical and Experimental Pharmacology and Physiology (2014) 41,371-380. PMID: 24754528 DOI: 10.1111/1440-1681.12222

6. Silva RT, Macedo de Resende L, Marco Aurélio Rocha Santos Ocular vestibular evoked myogenic potential: literature review. Audiology Communication Research 2016, vol.21, Dec 08, 2016. ISSN 2317- 6431. http://dx.doi.org/10.1590/2317-6431-2015-1651

7. Colebatch JG, Halmagyi GM, Skuse NF, Myogenic potentials generated by a click-evoked Vestibulocollic reflex. J Neurol Neurosurg Psychiatry (1994)57: 190-1997. doi: 10.1136/jnnp.57.2.190

8 M. Milkov, Applications of vestibular -evoked myogenic potentsials in vestibology. ORL International buletin № 2 2018, 34-40

9 Пламен Недев, Марио Милков, Клинична аудиология. ИК „Стено“, 2015, Медицински университет - Bapна, ISBN 978-619-7137-64-4; Издателска къща СТЕHО, ISBN 978-954-449-831-3 